\title{
Campo de conocimiento profesional que demandan los empleadores de la ciudad de Loja
}

\section{Field of professional knowledge demanded by employers in the city of Loja}

Namicela Toledo Mercy Yolanda

Castro Aguirre Isaac Sebastian

Calle Celi Juan Carlos

Universidad Internacional del Ecuador, Ecuador

Autor para correspondencia:menamicelato@uide.edu.ec, isaacastro171202@gmail.com, junkklle1@gmail.com

Fecha de recepción: 01 de Junio de 2017 - Fecha de aceptación: 15 de Agosto de 2017

Resumen: El presente estudio a empleadores se realiza con el objetivo de conocer el campo de conocimiento profesional que actualmente demanda el mercado laboral de la ciudad de Loja, cuyos resultados permiten evaluar la pertinencia de la oferta formativa de la Universidad Internacional del Ecuador- extensión Loja, y con ello dar un feedback al sector empresarial; para contribuir al mejoramiento de la empleabilidad de sus graduados. La muestra está conformada por 269 empleadores de la ciudad de Loja, resultado del cálculo del tamaño de la muestra con una población de 1650 empresas datos otorgados por el Servicio de Rentas Internas y un margen de error de 6\%, la técnica de investigación empleada es la encuesta, aplicada a través de un cuestionario estructurado con preguntas abiertas, cerradas y de opción múltiple, para su implementación se consideró el muestreo intencional no probabilístico tomando en cuenta la proporcionalidad de las empresas según su actividad y participación en el mercado, la cual fue aplicada puerta a puerta.. Los resultados permiten concluir que más del $50 \%$ de las empresas demandan carreras que se encuentran dentro del campo de conocimiento específico de Ciencias Sociales y del Comportamiento; y de Educación Comercial y Administración; y un mínimo porcentaje demandan carreras dentro de la Arquitectura y construcción; y, del campo de la Salud. Existiendo relación con la actividad económica comercial del sector empresarial de la ciudad de Loja. La oferta académica actual de la Universidad Internacional del Ecuador-extensión Loja se enmarca dentro del requerimiento de los empleadores.

Palabras clave: demanda; empresa; conocimiento; profesión; educación

Abstract: The present study to employers is carried out with the objective of knowing the field of professional knowledge that currently demands the labor market of the city of Loja, whose results allow to evaluate the pertinence of the training offer of the International University of Ecuador Loja extension, and Thereby giving feedback to the business sector; To contribute to the improvement of the employability of its graduates. The sample is made up of 269 employers from the city of Loja, resulting from the calculation of the size of the sample with a population of 1650 companies data provided by the Internal Revenue Service and a margin of error of 6\%, the research technique employed is The survey, applied through a questionnaire structured with open, closed and multiple choice questions, was considered for non-probabilistic intentional sampling taking into account the proportionality of the companies according to their activity and participation in 
the market, which was Applied door to door .. The results allow to conclude that more than 50\% of companies demand careers that are within the specific field of knowledge of Social and Behavioral Sciences; And Commercial Education and Administration; And a minimum percentage demand careers within Architecture and construction; And, the field of Health. Existing relationship with the commercial economic activity of the business sector of the city of Loja. The current academic offer of the International University of Ecuador-Loja extension is framed within the requirement of the employers.

Key words: demand; company; knowledge; profession; education

\section{Introducción}

La base fundamental para la oferta académica de las instituciones de educación superior es la pertinencia de cada carrera, de acuerdo al artículo 107 (Ley Orgánica de Educación Superior, 2010) el principio de pertinencia consiste en que la educación superior responda a las expectativas y necesidades de la sociedad, a la planificación nacional, y al régimen de desarrollo, a la prospectiva de desarrollo científico, humanístico y tecnológico mundial, y a la diversidad cultural (...).

Desde una visión global todos los sectores que mueven la economía de un país deben basar sus estrategias en un empoderamiento de desarrollo y crecimiento, la educación superior debe concebir que los profesionales que forman son el motor del progreso y del cambio, para ello debe adaptar la enseñanza a la evolución y al cambio provocado por la dinámica del mercado (Villalobos Monroy \& Pedroza Flores, 2009).

En la planificación nacional de ese país se consideran ejes importantes de trasformación orientados a garantizar el sumak kausay o buen vivir, en donde la participación del conocimiento y talento humano se va incrementando de forma gradual, por lo tanto, se requiere de una mejora en la calidad y pertinencia del sistema educativo en todos sus niveles, para que los profesionales estén preparados para responder a los requerimientos del mercado (Secretaría Nacional de Planificación y Desarrollo - Senplades, 2013).

De acuerdo a estudios realizados en el Ecuador se determina que la mayoría del empleo se agrupa en las ramas de servicios $(39,4 \%)$, comercio $(24,2 \%)$ y manufactura $(17,9 \%)$ (CensosINEC, Instituto Nacional de Estadísticas y Censos, 2016), la provincia de Loja al año 2015 registro una participación nacional del sector empresarial del 3.2\% (Censos-INEC, Instituto Nacional de Estadísticas y Censos, 2015), valores que la ubican en el décimo lugar del total de 24 provincias del país.

En la ciudad de Loja existen tres Universidades representativas que forman profesionales en diferentes campos del conocimiento (Consejo de Educación Superior-CES, 2016). La Universidad Internacional del Ecuador-extensión Loja en los últimos dos años ha graduado a 155 profesionales en los nueve campos específicos de conocimiento que oferta. De los cuales, anualmente se registran ciertos porcentajes de desempleados o empleados cuyas actividades están indirectamente relacionados al campo de su formación. Esta entidad, no cuenta con el acervo de conocimiento necesario sobre el aporte que brinda actualmente a través de la oferta académica y formación profesional a las necesidades del mercado laboral y al desarrollo de la matriz productiva local, regional y nacional. 
La UIDE-Loja consciente que la participación de los empleadores en los procesos de educación universitaria permite desarrollar la evaluación y planeación curricular, de forma tal que los programas educativos sean pertinentes y respondan a las exigencias del mercado de trabajo y la sociedad (Damián Simón, Montes Pauda, \& Arellano Mont, 2010); realiza el presente estudio de empleadores con el objetivo de identificar el campo de conocimiento que demanda actualmente el mercado laboral de la ciudad de Loja, cuyos resultados coadyuvaran a evaluar la pertinencia de sus carreras y mejorar su oferta académica, y con ello dar un feedback al sector empresarial. Con los resultados del estudio se comprueba la hipótesis que la oferta académica de la Universidad Internacional del Ecuador extensión Loja si responde a la demanda profesional del mercado Laboral de la ciudad de Loja.

\section{Metodología}

\section{Participantes}

Participaron en el presente estudio fuentes primarias: 269 empresarios de la población representada por 1650 empresas del sector público y privado de servicios, producción, y comercio de la ciudad de Loja. Y fuentes secundarias: la Base de datos otorgada por el Servicio de Rentas Internas de la Región Sur del Ecuador, con datos actualizados hasta el mes de septiembre del año 2016.

\section{Instrumentos}

El instrumento de investigación fue el cuestionario aplicado a través de una encuesta a empleadores de la ciudad de Loja, las preguntas fueron elaboradas en orden lógico y escritas de manera clara y sencilla, de tipo abiertas, cerradas y de opción múltiple; está estructurado por 12 ítems, que se dividen en dos partes; la primera contiene datos generales de la empresa; y la segunda los requerimientos y realidades en el sector empresarial. También existen preguntas estructuradas en la escala tipo Likert de 1 a 5 puntos (Muy importante, Importante, Poco importante, Nada importante).

\section{Procedimiento:}

Para determinar el segmento de la población a investigar se aplicó la fórmula del tamaño de la muestra, dando como resultado que se deben aplicar 269 encuestas, para la aplicación del cuestionario de la técnica investigativa seleccionada se aplicó el muestreo intencional considerando la proporcionalidad de las empresas según su actividad y participación en el mercado, una muestra representativa y útil que refleje homogeneidad y características de la población (Cruz del Castillo, Olivares Orozco, \& González García, 2014, p. 108)

Las encuestas fueron aplicadas en forma física, a través de la técnica puerta a puerta (McDaniel \& Gates, 2011), a los gerentes, directores de Talento Humano, o personal encargado de la administración y contratación de personal; los resultados fueron ingresados en el Paquete Estadístico para las Ciencias Sociales-SPSS lo cual facilito el análisis de las variables. 


\section{Resultados}

Tabla 1. Relación Entre El Tipo De Empresa Y La Actividad Económica Que Realiza.

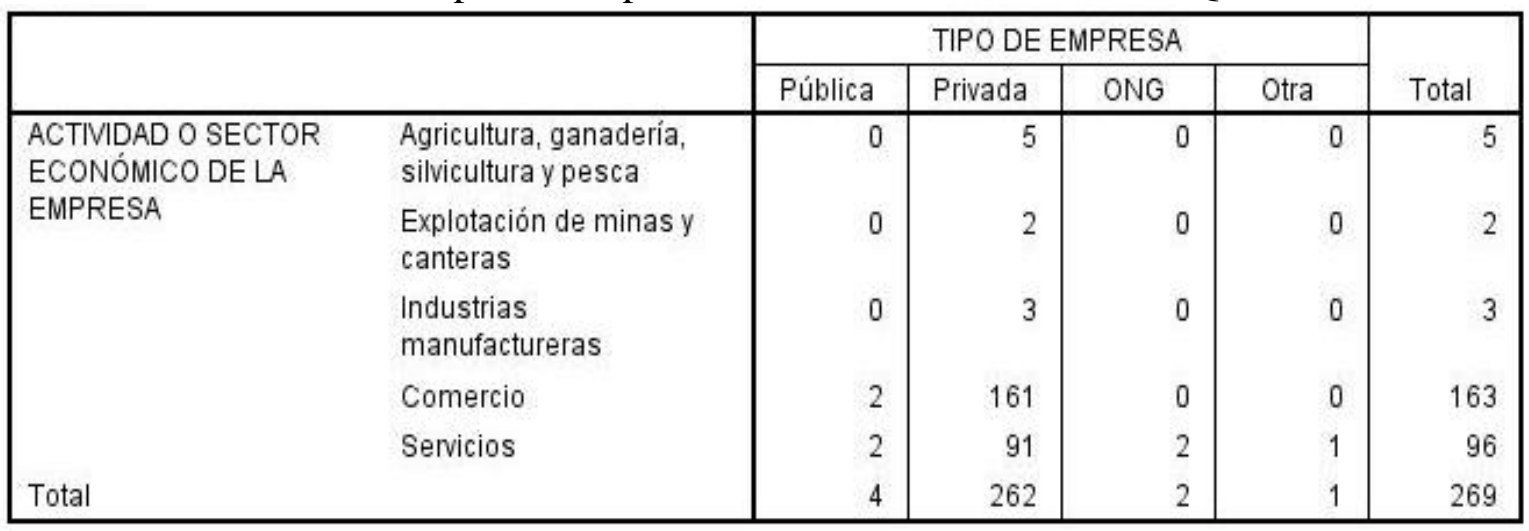

Fuente: Encuestas empleadores, Loja, 2016

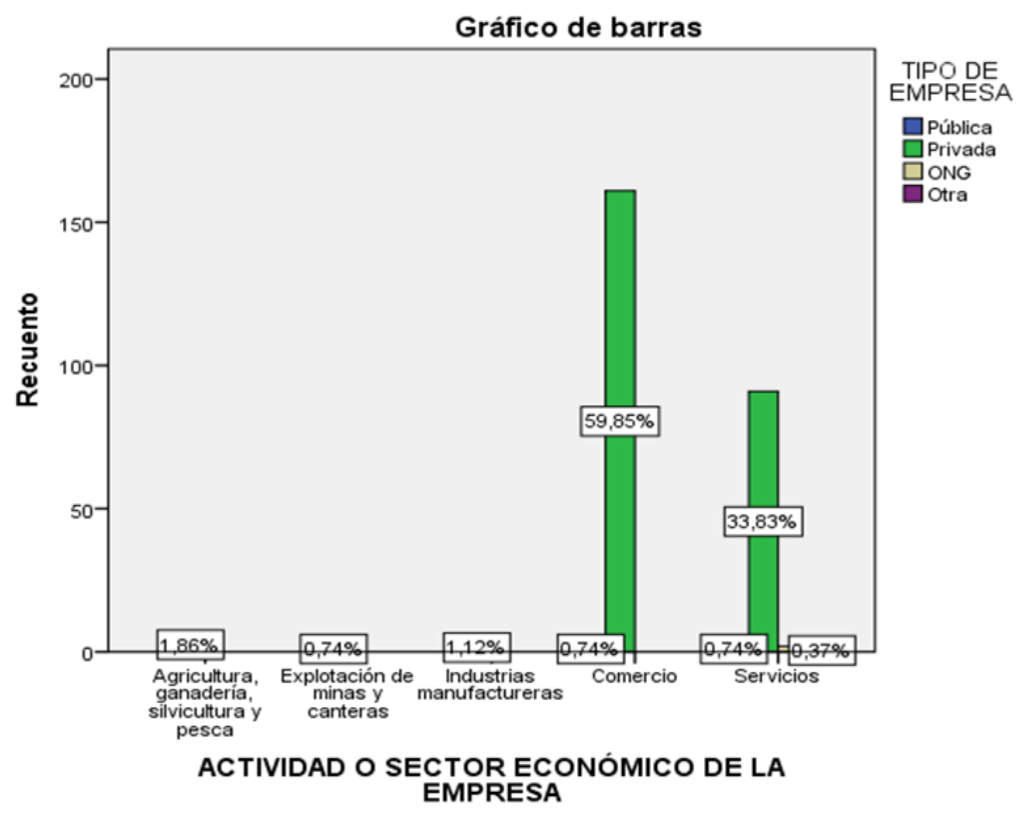

De las 269 empresas de la ciudad de Loja que corresponde a la muestra seleccionada, se encuestan a 262 empresas privadas, 4 entidades públicas, 2 ONG, y una que se encuentra fuera de este grupo; los sectores económicos a los que pertenecen son: 163 al Comercio que corresponde al 60\% de las empresas privadas; 96 a Servicios que equivale a 34\% de las empresas privadas; 5 Agricultura, ganadería, silvicultura y pesca; 3 Industrias y manufacturas; y; 2 Explotación de minas y canteras. Con estos datos se demuestra que los resultados incluyen la opinión de todos los sectores económicos de las empresas Lojanas.

Tabla 2. Campos De Los Profesionales Universitarios Que Requieren Las Empresas De La Ciudad De Loja 


\begin{tabular}{|c|c|c|c|c|}
\hline & & \multicolumn{2}{|c|}{ Respuestas } & \multirow{2}{*}{$\begin{array}{c}\text { Porcentaje de } \\
\text { casos }\end{array}$} \\
\hline & & $\mathrm{N}^{\circ}$ & Porcentaje & \\
\hline \multirow[t]{10}{*}{ Carreras $^{a}$} & Derecho & 44 & $10,2 \%$ & $16,4 \%$ \\
\hline & Economía & 18 & $4,2 \%$ & $6,7 \%$ \\
\hline & Finanzas & 33 & $7,7 \%$ & $12,3 \%$ \\
\hline & $\begin{array}{l}\text { Administración de } \\
\text { empresas }\end{array}$ & 108 & $25,1 \%$ & $40,1 \%$ \\
\hline & Mercadotecnia & 69 & $16,0 \%$ & $25,7 \%$ \\
\hline & Turismo & 27 & $6,3 \%$ & $10,0 \%$ \\
\hline & Arquitectura & 27 & $6,3 \%$ & $10,0 \%$ \\
\hline & Comercio & 51 & $11,9 \%$ & $19,0 \%$ \\
\hline & $\begin{array}{l}\text { Tecnologías de la } \\
\text { información }\end{array}$ & 31 & $7,2 \%$ & $11,5 \%$ \\
\hline & Otras & 22 & $5,1 \%$ & $8,2 \%$ \\
\hline Total & & 430 & $100,0 \%$ & $159,9 \%$ \\
\hline
\end{tabular}

a. Agrupación

Fuente: Encuestas empleadores, Loja, 2016

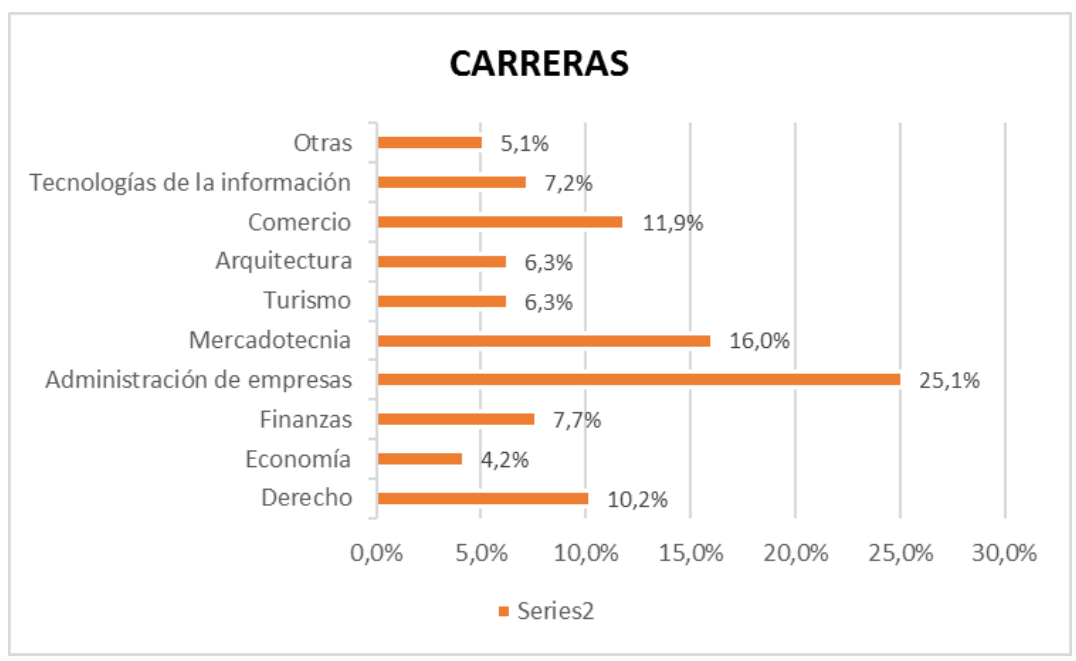

El requerimiento de profesionales universitarios que demandan las empresas de la ciudad de Loja para sus operaciones, se enmarcan en los siguiente campos del conocimiento de acuerdo a la denominación de carreras del (Consejo de Educación Superior-CES, 2016) : el 25\% en Administración de Empresas, el 16\% en Mercadotecnia, el 11.9\% Comercio, 10.2\% en Derecho, el 7.7\% en Finanzas, el 7.2\% en Tecnologías de la Información, el 6.3\% en Arquitectura y Turismo, respectivamente; el 5.1\% en otras carreras como Química-Biología, Gastronomía, etc., y, el $4.2 \%$ profesionales en Economía. Estos resultados se explican por el tipo de empresas que existen en la ciudad de Loja, ya que la mayoría se dedican al comercio, y necesitan profesionales para su administración.

Tabla 3. Competencias Profesionales Para El Desempeño Eficazmente En Su Campo Laboral 


\begin{tabular}{|c|c|c|c|c|c|c|c|c|c|c|}
\hline \multirow{2}{*}{$\begin{array}{c}\text { Competencias del profesional para el desempeño } \\
\text { eficaz en su campo laboral }\end{array}$} & \multicolumn{2}{|c|}{ Muy importante } & \multicolumn{2}{|c|}{ Importante } & \multicolumn{2}{|c|}{ Poco importante } & \multicolumn{2}{|c|}{ Nada importante } & \multicolumn{2}{|c|}{ Total general } \\
\hline & Respuestas & $\%$ & Respuestas & $\%$ & Respuestas & $\%$ & Respuestas & $\%$ & Respuestas & $\%$ \\
\hline Facilidad de expresión oral o escrita & 101 & $49 \%$ & 77 & $37 \%$ & 29 & $14 \%$ & 1 & $0 \%$ & 208 & $100 \%$ \\
\hline Dominio de su área o disciplina académica & 136 & $31 \%$ & 200 & $46 \%$ & 96 & $22 \%$ & 4 & $1 \%$ & 436 & $100 \%$ \\
\hline Compromiso ético & 177 & $43 \%$ & 167 & $41 \%$ & 60 & $15 \%$ & 4 & $1 \%$ & 408 & $100 \%$ \\
\hline Capacidad para diagnosticar problemas y resolverlos & 193 & $41 \%$ & 197 & $41 \%$ & 82 & $17 \%$ & 4 & $1 \%$ & 476 & $100 \%$ \\
\hline Capacidad para adquirir y aplicar nuevos conocimientos & 191 & $43 \%$ & 183 & $41 \%$ & 72 & $16 \%$ & 3 & $1 \%$ & 449 & $100 \%$ \\
\hline Capacidad de trabajar en equipo & 180 & $42 \%$ & 183 & $42 \%$ & 65 & $15 \%$ & 4 & $1 \%$ & 432 & $100 \%$ \\
\hline Compromiso con la empresa & 168 & $43 \%$ & 162 & $41 \%$ & 62 & $16 \%$ & 1 & $0 \%$ & 393 & $100 \%$ \\
\hline Responsabilidad & 170 & $43 \%$ & 158 & $40 \%$ & 63 & $16 \%$ & 4 & $1 \%$ & 395 & $100 \%$ \\
\hline Liderazgo & 181 & $42 \%$ & 176 & $41 \%$ & 72 & $17 \%$ & 3 & $1 \%$ & 432 & $100 \%$ \\
\hline Compañerismo & 180 & $44 \%$ & 167 & $40 \%$ & 62 & $15 \%$ & 4 & $1 \%$ & 413 & $100 \%$ \\
\hline Creatividad & 183 & $42 \%$ & 185 & $42 \%$ & 67 & $15 \%$ & 4 & $1 \%$ & 439 & $100 \%$ \\
\hline Crítico y autocrítico & 196 & $41 \%$ & 202 & $43 \%$ & 73 & $15 \%$ & 4 & $1 \%$ & 475 & $100 \%$ \\
\hline Capacidad de manejo de las TIC'S & 255 & $42 \%$ & 255 & $42 \%$ & 87 & $14 \%$ & 4 & $1 \%$ & 601 & $100 \%$ \\
\hline
\end{tabular}

Fuente: Encuestas empleadores, Loja, 2016

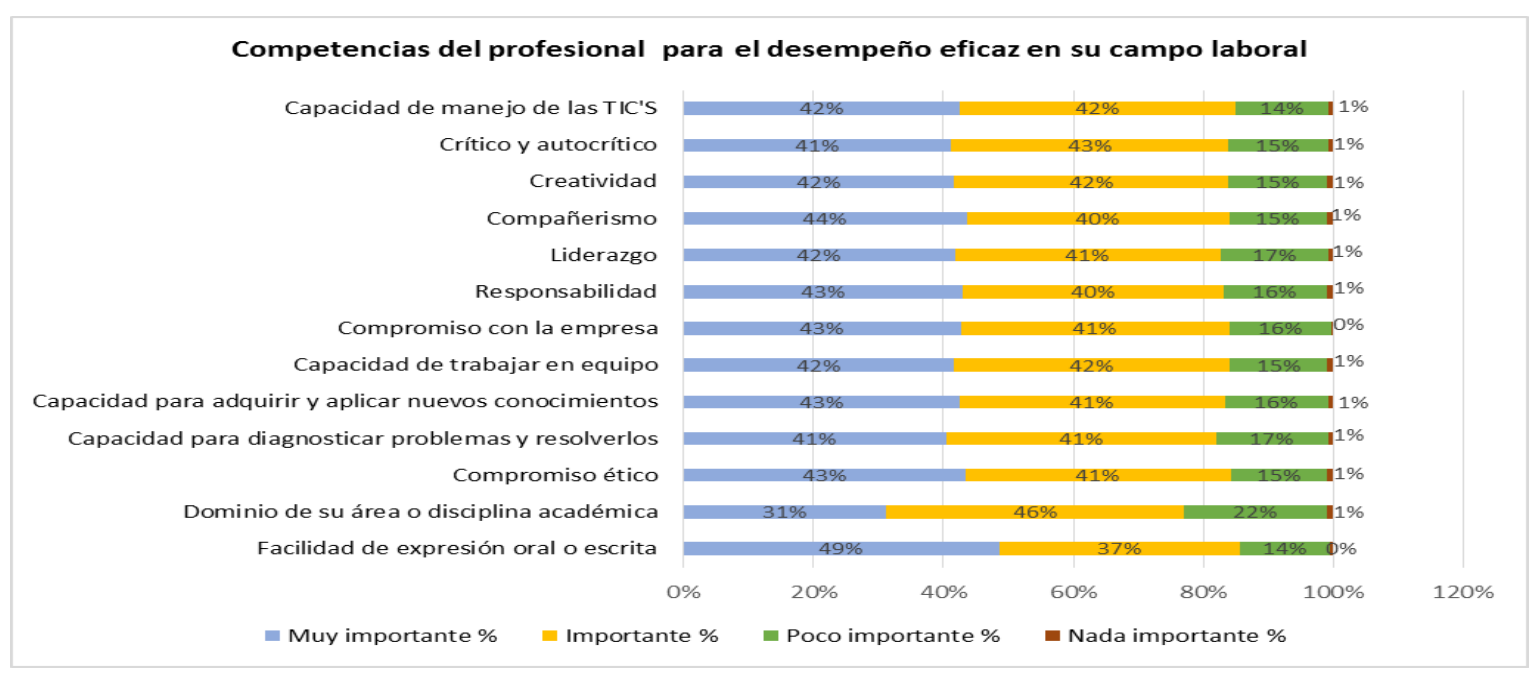

La mayoría de los empresarios de la ciudad de Loja consideran que es muy importante que los graduados cuenten con las siguientes competencias profesionales para que se desempeñen eficazmente en su campo profesional: facilidad de expresión oral o escrita, compañerismo, responsabilidad, compromiso con la empresa, capacidad para adquirir y aplicar nuevos conocimientos, compromiso ético, capacidad de manejo de las TIC's, creatividad, liderazgo, capacidad de trabajar en equipo, respectivamente. Lo que denota que los valores éticos y morales son muy importantes en la formación profesional, como lo sostiene (Rokeach, 1973; Schwartz, 1992, 2006) la educación en valores ha sido y es un tema de constante interés en el ámbito educativo y social, citado en (Morales, Trianes, \& Infante, 2013).

\section{Discusión}

Los tres campos profesionales que mayor demandan los empleadores del sector público y privado de la ciudad de Loja son: Administración de Empresas, Mercadotecnia y Comercio. Con estos resultados se comprueba la hipótesis que la oferta académica de la Universidad Internacional del Ecuador extensión Loja si responde a la demanda profesional del mercado Laboral de la ciudad de Loja. Haciendo una relación entre ésta demanda y los resultados de los estudios de seguimiento a graduados realizados en la UIDE-Loja, se determina que el nivel de desempleo de los graduados durante el año 2016 en la carrera de Ingeniería Comercial que de 
acuerdo al rediseño de carreras sería Administración de Empresas (Consejo de Educación Superior-CES, 2016), es del 14\% del total de la población encuestada; en la carrera de Marketing no existen graduados desempleados; (Universidad Internacional del Ecuador-extensión LojaUIDE-Loja, 2017).

Los profesionales en cualquiera de los campos del conocimiento deben ser competentes (dominio de la acción) y tener competencias (tener recursos), saber utilizar bien los recursossaberes en contextos particulares (Tejada Fernandez \& Ruiz Bueno, 2016). La formación y fortalecimiento de las competencias profesionales que demandan los empleadores, como la facilidad de expresión oral o escrita, compañerismo, responsabilidad, compromiso con la empresa, capacidad para adquirir y aplicar nuevos conocimientos, y compromiso ético, las carreras deben considerar como un requisito sine qua non para la formación de los estudiantes, con el fin de garantizar el mejoramiento de la empleabilidad de los graduados; brindar una educación acorde a las exigencias del mercado que permita orientar hacia el crecimiento económico y el desarrollo social de una sociedad, de acuerdo a (OCDE/CEPAL/CAF, 2017) cuanto mejor formados están los jóvenes, más posibilidades tienen de conseguir un empleo. La Universidad Internacional del Ecuador - extensión Loja en su malla curricular contempla asignaturas que permiten el fortalecimiento de éstas competencias.

\section{Conclusiones}

Las tres áreas de formación que más demandan los empresarios en la ciudad Loja son: Administración de Empresas, Mercadotecnia, y Comercio. Relacionando esta demanda con la realidad de la UIDE-Loja, de acuerdo al estudio de seguimiento a graduados del año 2017, se determina que el 86\% de la carrera de Ingeniería Comercial (Administración de Empresas) corresponde a la población económicamente activa y el 14\% de graduados está desempleada; en la carrera de Marketing no existen graduados desempleados, es decir, son empleados y cuentan con emprendimientos; mientras que el $73 \%$ de los graduados en la carrera de Negocios Internacionales (Comercio) están ocupadas y el $27 \%$ se encuentran desempleados. Lo cual permite evidenciar que el mercado Lojano responde a este tipo de requerimiento de profesionales.

Las principales competencias profesionales adicionales al perfil profesional que deben tener los graduados para que puedan desarrollar de manera eficiente su trabajo, de acuerdo al criterio de los empleadores, son: facilidad de expresión oral o escrita, compañerismo, responsabilidad, compromiso con la empresa, capacidad para adquirir y aplicar nuevos conocimientos, y compromiso ético.

\section{Bibliografía}

Censos-INEC, Instituto Nacional de Estadísticas y Censos. (2015). Directorio de Empresas. $\begin{array}{llllll}\text { Recuperado el } & 06 & \text { de } & \text { Febrero de }\end{array}$ http://www.ecuadorencifras.gob.ec/directoriodeempresas/

Censos-INEC, Instituto Nacional de Estadísticas y Censos. (2016). Panorama Laboral y Empresarial del Ecuador. Ecuador. Obtenido de http://www.ecuadorencifras.gob.ec/biblioteca/ 
Consejo de Educación Superior-CES. (2016). Reglamento de armonización de la nomenclatura de títulos Profesionales y grados académicos que confieren las instituciones de Educación Superior del Ecuador. Ecuador: RPC-SO-27-N² 289-2014.

Cruz del Castillo, C., Olivares Orozco, S., \& González García, M. (2014). Metodología de la Investigación. México: Larousse - Grupo Editorial Patria. Obtenido de www.ebrary.com

Damián Simón, J., Montes Pauda, E., \& Arellano Mont, L. J. (2010). Los estudios de opinión de empleadores. Estrategia para elevar la calidad de la educación superior no universitaria. REICE. Revista Iberoamericana sobre Calidad, Eficacia y Cambio en Educación, 179-203.

Ley Orgánica de Educación Superior. (2010). Ley Orgánica de Educación Superior. Ecuador. Obtenido de http://www.ces.gob.ec/index.php?option=com_phocadownload \&view=file \&id=651\&Item $\mathrm{id}=564$

McDaniel, C. J., \& Gates, R. (2011). Investigación de mercados. México: Cengage Learning.

Morales, F. M., Trianes, M. V., \& Infante, L. (2013). Perfiles de valores éticos en estudiantes universitarios. Aula Abierta, 41(2), 55-56.

OCDE/CEPAL/CAF. (2017). Perspectivas económicas de América Latina 2017: Juventud, competencias y emprendimiento. Paris: OECD Publishing. Obtenido de http://dx.doi.org/10.1787/leo-2017-es

Secretaría Nacional de Planificación y Desarrollo - Senplades. (2013). Plan Nacional del Buen vivir 2013-2017. Obtenido de http://www.buenvivir.gob.ec/

Tejada Fernandez , J., \& Ruiz Bueno, C. (2016). Evaluación de competencias profesionales en educación superior: retos e implicaciones. Educación, 19(1), 17-38.

Universidad Internacional del Ecuador-extensión Loja- UIDE-Loja. (2017). Estudio de Seguimiento a Graduados. Loja.

Villalobos Monroy, G., \& Pedroza Flores, R. (2009). Perspectiva de la teoría del capital humano acerca de la relación entre educación y desarrollo económic. Tiempo de Educar, pp. 273306. Obtenido de http://www.redalyc.org/articulo.oa?id=31112987002 